Jacqueline Rodrigues Longchamps

O modo verbal na aquisição do Português Brasileiro: evidências naturalistas e experimentais da percepção, expressão e compreensão da distinção realis/irrealis

Dissertação de Mestrado

Dissertação apresentada ao Programa de Pósgraduação em Letras da PUC-Rio como requisito parcial para obtenção do título de Mestre em Letras.

Orientadora: Prof ${ }^{\mathrm{a}} \mathrm{Dr}^{\mathrm{a}}$ Letícia Maria Sicuro Corrêa 
Jacqueline Rodrigues Longchamps

\begin{abstract}
O modo verbal na aquisição do Português Brasileiro: evidências naturalistas e experimentais da percepção, expressão e compreensão da distinção realis/irrealis
\end{abstract}

Dissertação apresentada como requisito parcial para obtenção do grau de Mestre pelo Programa de Pós-Graduação em Letras do Departamento de Letras do Centro de Teologia e Ciências Humanas da PUC-Rio. Aprovada pela Comissão Examinadora abaixo assinada.

Profa . Letícia Maria Sicuro Corrêa Orientadora Departamento de Letras - PUC-Rio

Profa. Eneida do Rêgo Monteiro Bomfim Departamento de Letras - PUC-Rio

Profa. Ana Paula Scher

USP

Prof. Paulo Fernando Carneiro de Andrade Coordenador Setorial do Centro de Teologia e Ciências Humanas - PUC-Rio

Rio de Janeiro, 27 de março de 2009. 
Todos os direitos reservados. É proibida a reprodução total ou parcial do trabalho sem autorização da universidade, da autora e da orientadora.

\section{Jacqueline Rodrigues Longchamps}

Graduou-se no Bacharelado em Letras pela Universidade Estácio de Sá em 2006. Obteve título de Licenciada em Letras pela mesma Instituição em 2006. Concluiu, em 2009, o Mestrado em Letras (área de concentração: Estudos da Linguagem) na PUC-Rio. Atua no Grupo de Pesquisa do LAPAL - Laboratório de Psicolingüística e Aquisição da Linguagem, vinculado ao Departamento de Letras - PUCRio. Áreas de interesse: Psicolingüística, Aquisição da Linguagem, Déficits da Linguagem, Sintaxe Gerativa.

Ficha Catalográfica

Longchamps, Jacqueline Rodrigues

O modo verbal na aquisição do português brasileiro : evidências naturalistas e experimentais da percepção, expressão e compreensão da distinção realis/irrealis / Jacqueline Rodrigues Longchamps ; orientadora Letícia Maria Sicuro Corrêa. - 2009.

149 f. : il. (color.) ; $30 \mathrm{~cm}$

Dissertação (Mestrado em Letras)-Pontifícia Universidade Católica do Rio de Janeiro, Rio de Janeiro, 2009.

Inclui bibliografia

1. Letras - Teses. 2. Aquisição da linguagem. 3. Modo verbal. 4. Categorias funcionais. 5. Interfaces fônica e semântica. 6. Hipótese da oposição semântica. 7. Bootstrapping. I. Corrêa, Letícia Maria Sicuro. II. Pontifícia Universidade Católica do Rio de Janeiro. Departamento de Letras. III. Título. 
Para meus pais, Cid (in memoriam) e Dina, a quem devo tudo o que sou. 


\section{Agradecimentos}

À Professora Letícia, exemplo de profissionalismo, pela orientação, pelo apoio, a atenção, o carinho e, sobretudo, pela confiança em meu trabalho. Minha gratidão, meu respeito e minha admiração: sempre.

Às Professoras que participaram da Comissão Examinadora.

Ao Grupo de Pesquisa em Processamento e Aquisição da Linguagem (GPPAL/LAPAL), pela acolhida e pelos ensinamentos preciosos.

A todos os meus Professores da PUC-Rio, pela inestimável contribuição à minha formação.

A todos os colegas do LAPAL, pelo convívio alegre e sadio, pelos comentários, sugestões, apoio e amizade.

À Mercedes Marcilese, pela ajuda e incentivo quanto aos primeiros experimentos.

Às Creches Criativa, Jardim dos Pirilampos, Tabladinho e ao Colégio Stockler por cederem gentilmente suas dependências para a realização dos testes.

Às crianças que participaram dos experimentos, sem as quais este trabalho não teria sido possível.

À PUC-Rio, à CAPES e à FAPERJ pelos auxílios concedidos.

A todos que contribuíram de alguma forma para a realização deste projeto.

À minha mãe, pelo apoio em todos os sentidos e pela infinita compreensão para com minha "ausência". 


\section{Resumo}

Longchamps, Jacqueline Rodrigues; Corrêa, Letícia Maria Sicuro (Orientadora). O modo verbal na aquisição do Português Brasileiro: evidências naturalistas e experimentais da percepção, expressão e compreensão da distinção realis/irrealis. Rio de Janeiro, 2009. 149 p. Dissertação de Mestrado - Departamento de Letras, Pontifícia Universidade Católica do Rio de Janeiro.

A presente dissertação se insere em uma teoria da aquisição da linguagem que concilia uma abordagem psicolingüística com uma concepção minimalista de língua. As hipóteses de trabalho que a nortearam foram: o que é gramaticalmente relevante tem de estar expresso na interface fônica; a interpretação semântica do complexo TAM (Tempo, Aspecto, Modo) acarreta dificuldade na identificação da morfologia de modo; distinções conceptuais fundamentais, como a oposição realis/irrealis, são expressas pela criança, independentemente de a morfologia da língua ter sido totalmente identificada (Hipótese da Oposição Semântica). Um estudo da produção espontânea de duas crianças de 1;5 a 2;5 de idade e 4 experimentos foram conduzidos. $\mathrm{Na}$ análise dos dados da fala espontânea, constatou-se que a oposição realis/irrealis é expressa pela criança por meio da distinção entre formas verbais flexionadas e não-flexionadas, sendo que o chamado Infinitivo Raiz (IR) aparece como alternativa ao subjuntivo para expressão do modo irrealis. Os resultados de dois experimentos de produção eliciada sugerem que a distinção indicativo/subjuntivo é percebida por crianças de 3 anos, embora aos 5 anos de idade a expressão do subjuntivo ainda apresente dificuldade. Um experimento de compreensão com perguntas SIM/NÃO com crianças de 5-7 anos, e seu follow-up, sugerem que o contraste indicativo/subjuntivo em completivas é reconhecido, ainda que respostas negativas imponham dificuldades que independem do modo, particularmente aos 5 anos. Os resultados são compatíveis com as hipóteses e sugerem que a presença do IR no português brasileiro é indicativa da alteração paramétrica em curso (de sujeito nulo para preenchido).

\section{Palavras-chave}

Aquisição da linguagem; modo verbal; categorias funcionais; interfaces fônica e semântica; Hipótese da Oposição Semântica; bootstrapping. 


\section{Abstract}

Longchamps, Jacqueline Rodrigues; Corrêa, Letícia Maria Sicuro (Advisor).

The acquisition of verbal mood in Brazilian Portuguese: naturalist and experimental evidence of the perception, expression and comprehension of the realis/irrealis mood distinction. Rio de Janeiro, 2009. 149 p. MSc. Dissertation - Departamento de Letras, Pontifícia Universidade Católica do Rio de Janeiro.

This dissertation is in the context of a theory which reconciles a psycholinguist approach to language acquisition and a minimalist conception of language. The working hypotheses were: grammatically relevant information has to be legible at the phonetic interface; the semantic interpretation of the TAM complex (Tense, Aspect, Mood) poses difficulty to the identification of the verbal mood morphology; basic conceptual distinctions, such as the realis/irrealis contrast, are expressed by the child, no matter whether or not his/her language morphology has been totally identified (The Semantic Opposition Hypothesis). A study of the spontaneous speech production of two children, aged 1;5 to 2;5, and 4 experiments were carried out. The analysis of the production data revealed that the realis/irrealis opposition is expressed by the child by means of the distinction between inflected/uninflected verbal forms and the Root Infinitive (RI) represents an alternative form to the subjunctive for the expression of the irrealis mood. The results of two elicited production experiments suggest that the indicative/subjunctive distinction is perceived by 3-year-olds, although the expression of the subjunctive mood still poses difficulty even for 5-year-olds. A comprehension experiment involving YES/NO questions with 5-7-year-olds, and its follow-up, suggest that the indicative/subjunctive opposition in subordinate clauses is recognized, even though negative answers pose difficulties that are not related to verbal mood, particularly at 5 years of age. The results are compatible with the hypotheses, suggesting that the presence of RIs in Brazilian Portuguese indicates a parametric shift in course (from null subject to non-null subject).

\section{Keywords}

Language acquisition; verbal mood; functional categories; phonetic and semantic interfaces; Semantic Opposition Hypothesis; bootstrapping. 


\section{SUMÁRIO}

1 Introdução 13

1.1 Hipóteses de trabalho 15

1.2 Relevância da pesquisa $\quad 16$

$\begin{array}{ll}1.3 \text { Objetivos } & 17\end{array}$

$\begin{array}{ll}1.4 \text { Organização do trabalho } & 18\end{array}$

2 Modo verbal $\quad 19$

2.1 Modo e modalidade de um ponto de vista descritivo 19

2.1.1 Gramática tradicional 19

2.1.2 Lingüística descritiva 22

2.2 Modo na teoria lingüística gerativista 36

2.2.1 Quadro teórico gerativista 36

2.2.2 Concepção minimalista de língua 37

2.2.3 Modo como categoria funcional 39

$\begin{array}{ll}2.2 .4 \text { Categorias funcionais } & 40\end{array}$

2.3 Modo no português do Brasil $\quad 45$

3 Aquisição do modo verbal 53

3.1 Percepção de distinções gramaticais na interface fônica 53

3.2 Aquisição de modo: produção 56

4 Metodologia $\quad 71$

4.1 Metodologia naturalista: produção da fala espontânea 71

4.1.1 Estudo da produção da fala espontânea 72

4.2 Metodologia experimental $\quad 72$

4.2.1 Técnicas utilizadas nos experimentos 73

5 Estudo da manifestação de modo em produção espontânea 76

6 Experimentos de percepção e produção de modo 96

6.1 Experimento 1: Imitação eliciada 96 
7.1 Experimento 3: Compreensão do subjuntivo

7.2 Experimento 4 (follow up): Compreensão do indicativo (comparação realis/irrealis)

8 Conclusão

10.1 ENY - Seção 23 (como exemplo) 140

10.2 JES - Seção 23 (como exemplo) 143

11 Apêndice 2: Estímulos experimentais 145

11.1 Experimento $1 \quad 145$

11.2 Experimento $2 \quad 146$

11.3 Experimento $3 \quad 147$

11.4 Experimento $4 \quad 148$ 


\section{Lista de gráficos}

Gráfico 1 - Médias de respostas-alvo em função de idade (Experimento 1)

Gráfico 2 - Médias de respostas-alvo em função de modo (Experimento 1)

Gráfico 3 - Médias de respostas-alvo em função de idade e modo (Experimento 1)

Gráfico 4: Percentual de alternativas de substituição na condição subjuntivo (Experimento 1)

Gráfico 5: Médias de respostas-alvo em função de idade (Experimento 2)

Gráfico 6: Médias de respostas-alvo em função de forma verbal (Experimento 2)

Gráfico 7: Médias de respostas-alvo em função de interação entre idade e forma verbal (Experimento 2)

Gráfico 8: Percentual de substituições na condição subjuntivo (Experimento 2)

Gráfico 9: Médias de respostas-alvo SIM (Experimento 3)

Gráfico 10: Médias de respostas-alvo SIM em função de interação entre forma verbal e idade (Experimento 3)

Gráfico 11: Médias de respostas-alvo NÃO em função de idade (Experimento 3)

Gráfico 12: Médias de respostas-alvo SIM nos Experimentos 3 e 4 em função de idade

Gráfico 13: Médias de respostas-alvo NÃO nos Experimentos 3 e 4 em função de idade

Gráfico 14: Médias dos contrastes de sujeito e de tempo verbal (TV) em função de idade (Experimento 4) 


\section{Lista de quadros}

Quadro 1 - Comparação das desinências do imperativo e do subjuntivo no português (Cunha \& Cintra, 1985)

Quadro 2 - Hierarquia das projeções funcionais em IP

(Cinque, 1999)

Quadro 3 - Estrutura frasal segundo Rizzi (1997)

Quadro 4 - Combinações de traços de pessoa e de infinitivo (Perales et al., 2006)

Quadro 5 - Paradigmas de conjugação verbal no PB (antigo e atual)

Quadro 6 - Exemplos das produções de ENY e JES em contextos realis e irrealis

Quadro 7 - Exemplos de locuções produzidas por ENY e JES em contextos irrealis

Quadro 8 - Exemplos de estímulos e condições experimentais do Experimento 1

Quadro 9 - Exemplos de estímulos e condições experimentais do Experimento 2

Quadro 10 - Exemplos de estímulos e condições experimentais do Experimento 3

Quadro 11 - Exemplos de estímulos e perguntas do Experimento 4 para respostas SIM

Quadro 12 - Exemplos de estímulos e perguntas do Experimento 4 para respostas NÃO 


\section{Lista de tabelas}

Tabela 1 - Percentual de formas verbais produzidas por ENY e JES

Tabela 2 - Percentual de formas produzidas em contextos realis e irrealis por ENY e JES

Tabela 3 - Distribuição das locuções em função de contextos realis e irrealis

Tabela 4 - Percentual de respostas-alvo e respostas alternativas (Experimento 1)

Tabela 5 - Percentual de substituições na condição subjuntivo (Experimento 1)

Tabela 6 - Percentual de respostas-alvo e respostas alternativas na condição subjuntivo (Experimento 2)

Tabela 7 - Percentual de substituições na condição subjuntivo (Experimento 2)

Tabela 8 - Percentual de respostas-alvo e respostas alternativas na condição infinitivo (Experimento 2)

Tabela 9 - Teste-t: diferenças entre respostas SIM em função de condição experimental e de idade (Experimento 3)

Tabela 10 - Teste-t: diferenças entre respostas SIM e NÃO nos Experimentos 3 e 4

Tabela 11 - Diferenças entre as respostas NÃO em função dos contrastes de sujeito e de tempo (Experimento 4)

Tabela 12 - Percentual de crianças que utilizaram estratégias de respostas SIM /NÃO 